\title{
Glycyrrhizin affects monocyte migration and apoptosis by blocking HMGB1 signaling
}

\author{
JIA-YING TAN, FENG ZHAO, SHUI-XIANG DENG, HE-CHEN ZHU, YE GONG and WEI WANG \\ Department of Critical Care Medicine, Huashan Hospital, Fudan University, Shanghai 200040, P.R. China
}

Received July 8, 2017; Accepted January 29, 2018

DOI: $10.3892 / \mathrm{mmr} .2018 .8598$

\begin{abstract}
Monocytes serve an important role in systemic inflammation. High mobility group box-1 protein (HMGB1) promotes recruitment and suppresses apoptosis in monocytes through the receptor for advanced glycation end products/ nuclear factor (NF)- $\kappa \mathrm{B}$ and toll-like receptor 4/mitogen-activated protein kinase (MAPK)/extracellular signal-regulated kinase (ERK) signaling pathways. Glycyrrhizin (GL), an effective component of licorice, weakens the proinflammatory effect of HMGB1. The present study investigated the effect of GL on the migration and apoptosis of monocytes associated with HMGB1 signaling. THP-1 cells were used to evaluate the behavior of monocytes in response to GL treatment, and the downstream pathways were investigated. GL suppressed HMGB1-induced monocyte migration and increased HMGB1-inhibited monocyte apoptosis. GL inhibited the activation of the NF- $\mathrm{B}$ and MAPK/ERK signaling pathways induced by HMGB1 and decreased the expression of monocyte chemoattractant protein-1 (MCP-1) and myeloid cell leukemia 1 (Mcl-1). Taken together, the results indicated that GL may suppress the migration of monocytes and induce apoptosis to reduce systemic inflammation by blocking downstream NF-кB/MCP-1 and MAPK/ERK/Mcl-1 signaling pathways.
\end{abstract}

\section{Introduction}

Sepsis is defined as life-threatening organ dysfunction caused by a dysregulated host response to infection (1). It is a complex pathological process that leads to an inflammatory cascade within the host, and it is triggered by pathogen-associated molecular patterns caused by bacteria or other pathogens. Monocytes play an essential role in the initiation and maintenance of host

Correspondence to: Professor Ye Gong or Professor Wei Wang, Department of Critical Care Medicine, Huashan Hospital, Fudan University, 12 Middle Urumqi Road, Shanghai 200040, P.R. China

E-mail: drgongyeicu@163.com

E-mail: drwangwei3037@126.com

Key words: glycyrrhizin, monocyte, high mobility group box-1 protein, receptor for advanced glycation end products, nuclear factor- $\kappa \mathrm{B}$, toll-like receptor 4 , mitogen-activated protein kinase, monocyte chemoattractant protein-1, myeloid cell leukemia 1 responses in systemic inflammation (2). When an inflammatory reaction occurs, monocytes can aggregate at the site of inflammation and release a large number of inflammatory factors and chemokines such as interleukin (IL)-1b, IL-6 and tumor necrosis factor (TNF)- $\alpha$, which further intensify the inflammatory response. High mobility group box-1 protein (HMGB1) is a late mediator that is extensively involved in systemic and local inflammatory responses through receptor pathways such as the receptor for advanced glycation end products (RAGE) and toll-like receptors (TLR). HMGB1 promotes monocyte recruitment by activating the RAGE/nuclear factor (NF)- $\kappa$ B signaling pathway, and it inhibits monocyte apoptosis by activating the TLR4/mitogen-activated protein kinase (MAPK)/extracellular signal-regulated kinase (ERK) signaling pathway (3). Monocyte chemoattractant protein-1 (MCP-1) is a chemokine involved in the initiation and maintenance of the inflammatory response. Myeloid cell leukemia 1 (Mcl-1) is an anti-apoptotic protein. These two cytokines both play an important role in inflammation.

Glycyrrhizin (GL), also called glycyrrhizic acid, is an effective component of licorice. It possesses many pharmacological properties including antiviral, anti-inflammatory, anti-tumor, and hepatoprotective effects. In East Asia, GL has been clinically used for over two decades as an anti-inflammatory factor and for the treatment of chronic hepatitis (4-6). GL was reported to interact directly with HMGB1, thereby reducing the concentration of HMGB1 and inhibiting its biological activities (7). In addition, GL can weaken the pro-inflammatory effect of HMGB1 through different signaling pathways. Zhao et al (8) showed that interaction between cell receptors and HMGB1 could be blocked by GL, leading to the inactivation of downstream MAPKs/NF- $\kappa \mathrm{B}$ signaling pathways. A recent study showed that an appropriate decrease in the number of neutrophils and monocytes is beneficial for the survival of cecal ligation and puncture (CLP) mice (9). Although the important role of monocytes in sepsis is known, the effect of GL on the recruitment and apoptosis of monocytes to reduce the HMGB1-mediated inflammatory cascade remains unclear. In addition, the effects on monocytes in relation to the concentration of GL remain unexplored.

In this study, we first examined the effects of HMGB1 on promoting the migration and inhibiting apoptosis of monocytes. Next, we investigated the effects of GL treatment on HMGB1-affected monocytes using THP-1 cells. Finally, the mechanism underlying the effect of GL treatment on 
monocytes through the interplay between HMGB1 and downstream signaling pathways was investigated.

\section{Materials and methods}

Cell culture. THP-1 cells were purchased from the cell bank of the Chinese Academy of Sciences (Shanghai, China) and cultured in RPMI1640 medium (HyClone, Logan, UT, USA) containing $10 \%$ fetal bovine serum (FBS; Gibco; Thermo Fisher Scientific, Inc., Waltham, MA, USA) and 10x10 $10^{4} \mathrm{U} / \mathrm{ml}$ penicillin/streptomycin (HyClone). Cells were cultured in an incubator at $37^{\circ} \mathrm{C}$ with $5 \% \mathrm{CO}_{2}$ and $95 \% \mathrm{O}_{2}$.

Transwell assay. Transwell chambers used were ECM550 (Chemicon, Temecula, CA, USA). THP-1 cells with good growth conditions were made into single cell suspensions and $1 \times 10^{5}$ (total volume of $200 \mu \mathrm{l}$ ) cells were added to the upper chamber. A volume of $300 \mu \mathrm{l}$ of serum-containing culture medium with different concentrations of recombinant human HMGB1 (R\&D Systems, Inc., Minneapolis, MN, USA) was added to the lower chamber and incubated for $24 \mathrm{~h}$ at $37^{\circ} \mathrm{C}$. Then, the medium in the lower chamber was aspirated, sodium chloride-alcohol was added, and washed after fixing for 10 min. Then, $0.1 \%$ crystal violet (Sigma-Aldrich, St. Louis, MO, USA) was added to the lower chamber and stained for $10 \mathrm{~min}$. Cells on the membrane of the upper chamber were carefully wiped, while cells on the basement membrane of the lower chamber were counted in 6 view fields randomly selected under a $\mathrm{x} 200$ optical microscope. The average was recorded as the number of THP-1 cells passing through the artificial basement membrane. Different concentrations of GL (Sigma-Aldrich) were added to the culture fluid in the lower chamber as treatment before the $24 \mathrm{~h}$ culture.

Flow cytometric detection of cell apoptosis. Staurosporine (STS) $(300 \mathrm{nM})$ with or without different concentrations of HMGB1 was added to the THP-1 cell culture medium and incubated for $24 \mathrm{~h}$. After centrifugation and resuspension in PBS, $5-10 \times 10^{4}$ cells were stained with $200 \mu$ l Annexin V-fluorescein isothiocyanate (FITC) and $10 \mu \mathrm{l}$ propidium iodide (PI) following secondary centrifugation. Flow cytometry was performed by using a FACSCalibur system (Becton Dickinson, Franklin Lakes, NJ, USA) after incubation at room temperature in the dark for 10-20 min and ice bath. The STS and apoptosis detection kit Annexin V-FITC were obtained from Beyotime Institute of Biotechnology (Shanghai, China). The scatter plot was divided into four quadrants: The left lower quadrant was defined as viable cells ('low' FITC and 'low' PI signal), the right upper quadrant was defined as necrotic cells ('high' FITC and 'high' PI signal), and the right lower quadrant was defined as apoptotic cells ('high' FITC and 'low' PI signal). The percentage of Annexin V-positive cells in the total cells of the scatter plot was used to determine the results. Different concentrations of GL were added to the samples prior to the one day incubation for the intervention experiment.

Western blotting. Cellular proteins were extracted using lysis buffer, and the concentrations of protein were measured using a BCA Protein Assay kit (Thermo Fisher Scientific, Inc.). For the detection of NF- $\mathrm{B}$ level in nucleus, nuclear
Table I. Primer sequences for polymerase chain reactions.

\begin{tabular}{lll}
\hline Primer & Strand & \multicolumn{1}{c}{ Sequence (5'-3') } \\
\hline MCP-1 & Sense & CAGCCAGATGCAATCAATGCC \\
& Antisense & TGGAATCCTGAACCCACTTCT \\
\multirow{2}{*}{-actin } & Sense & CATGTACGTTGCTATCCAGGC \\
& Antisense & CTCCTTAATGTCACGCACGAT
\end{tabular}

MCP-1, monocyte chemoattractant protein-1.

protein was extracted using nuclear extraction kit (Epigentek, Farmingdale, NY, USA) in accordance with the manufacturer's instructions. Equal protein amounts were fractionated in 5-8\% SDS-PAGE gels and transferred to polyvinylidene fluoride (PVDF) membranes (Millipore, Billerica, MA, USA). After blocking with $5 \%$ skimmed milk powder, the membranes were incubated with the following primary antibodies at 1:1,000 dilution: Cleaved caspase-3, PARP, phosphorylation of I $\mathrm{B} \alpha$ (p-I $\mathrm{B} \alpha$ ), I $\mathrm{B} \alpha, \mathrm{NF}-\kappa \mathrm{B}$ p65, phosphorylation level of ERK1/2 (p-ERK1/2), ERK1/2, and Mcl-1 (all from Cell Signaling Technology, Beverly, MA, USA), and HMGB1, $\beta$-actin and Lamin B1 (all from Santa Cruz Biotechnology, Inc., Dallas, TX, USA) overnight at $4^{\circ} \mathrm{C}$. The membranes were then incubated with HRP-conjugated rabbit or mouse secondary antibodies (Cell Signaling Technology) at room temperature for $2 \mathrm{~h}$ after washing. Immunoblots were detected by using an enhanced chemiluminescence reagent (Tiangen Biotech, Co., Ltd., Beijing, China) and imager (Bio-Rad Laboratories, Inc., Hercules, CA, USA). Gray value was analyzed with the software ImageJ, and the gray coefficient ratio was calculated.

Reverse transcription-quantitative polymerase chain reaction $(R T-q P C R)$. Total RNA was isolated from THP-1 cells using the TRIzol reagent (Invitrogen; Thermo Fisher Scientific, Inc.). Reverse transcription was performed using a First Strand cDNA Synthesis kit (Fermentas, ON, Canada). PCR amplification was performed using a One Step SYBR-Green I Quant qRT-PCR kit (Tiangen Biotech, Co., Ltd.) on an ABI 7000 real-time PCR system (Applied Biosystems; Thermo Fisher Scientific, Inc.). Table I shows the primers used for MCP-1 and $\beta$-actin synthesized by Invitrogen (Thermo Fisher Scientific, Inc.) for PCR. PCR parameters were $5 \mathrm{~min}$ at $94^{\circ} \mathrm{C}$, followed by 40 cycles of $30 \mathrm{sec}$ at $94^{\circ} \mathrm{C}, 30 \mathrm{sec}$ annealing at $60^{\circ} \mathrm{C}$ for MCP-1 or $55^{\circ} \mathrm{C}$ for $\beta$-actin, and $30 \mathrm{sec}$ at $72^{\circ} \mathrm{C}$, ending at $72^{\circ} \mathrm{C}$ for $10 \mathrm{~min}$. The $2^{-\Delta \Delta \mathrm{Cq}}$ method (10) was used to calculate the relative levels of MCP-1 mRNA, and $\beta$-actin expression was used for normalization.

Statistical analysis. Data are expressed as the mean \pm SEM. Differences between groups were evaluated using one-way ANOVA followed by the Newman-Keuls test, using SPSS version 16.0 software (SPSS, Inc., Chicago, IL, USA). Statistical significance was established when $\mathrm{P}<0.05$.

\section{Results}

GL treatment suppresses HMGBl-induced monocyte migration. THP-1 cells were treated with different 
concentrations of HMGB1 $(0.1,0.5$, and $1 \mu \mathrm{g} / \mathrm{ml})$. The results of Transwell assays showed that HMGB1 significantly increased the number of migrated cells, especially at higher concentrations (Fig. 1A). GL at all concentrations tested (10, 50 , and $100 \mu \mathrm{g} / \mathrm{ml}$ ) suppressed the HMGB1-induced monocyte migration, particularly at 50 and $100 \mu \mathrm{g} / \mathrm{ml}$ (Fig. 1B).

GL treatment increases monocyte apoptosis. THP-1 cells were treated with STS and HMGB1 $(0.1,0.5$, and $1 \mu \mathrm{g} / \mathrm{ml})$. Fig. 2A shows that the control group had a lower apoptosis rate, and STS significantly induced apoptosis. HMGB1 at 0.5 and $1 \mu \mathrm{g} / \mathrm{ml}$ suppressed STS-induced apoptosis, and GL at 50 and $100 \mu \mathrm{g} / \mathrm{ml}$ significantly improved the inhibition of apoptosis by HMGB1 (Fig. 2B). Furthermore, we explored effects of HMGB1 and GL on key factors in apoptotic pathway. As shown in Fig. 2C and D, STS significantly increase the level of cleaved caspase-3 and PARP. HMGB1 suppressed the STS-induced cleavage of caspase-3 and PARP, and GL significantly increased the cleavage at 50 and $100 \mu \mathrm{g} / \mathrm{ml}$.

GL treatment inhibits $H M G B 1$ induced activation of $N F-\kappa B$ and downregulates $M C P-1$. HMGB1 promotes monocyte recruitment by activating the $\mathrm{RAGE} / \mathrm{NF}-\kappa \mathrm{B}$ signaling pathway (3). p-IкB $\alpha$ results in the activation of nuclear trans-

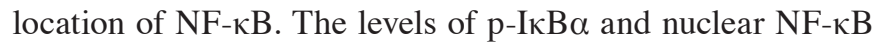
p65 were detected to evaluate the effect of GL treatment on the activation of NF- $\kappa B$. Fig. 3 shows that HMGB1 increased the level of $\mathrm{p}-\mathrm{I} \kappa \mathrm{B} \alpha$ and nuclear NF- $\mathrm{kB}$ p65, whereas GL decreased this level by suppressing the function of HMGB1. MCP-1 has the ability to recruit and activate particular leukocytes and is a potential downstream effector of NF- $\mathrm{kB}$ (11). RT-qPCR was performed to detect the effect of HMGB1, the NF- $\mathrm{kB}$ inhibitor QNZ (100 nM; Selleck Chemicals, Houston, TX, USA), and GL on the expression of MCP-1. HMGB1 significantly increased the mRNA expression of MCP-1, whereas QNZ and GL remarkably suppressed the induction of MCP-1 expression by HMGB1 (Fig. 4).

GL treatment inhibits HMGB1-induced activation of MAPK/ERK and downregulates Mcl-1. Since HMGB1 inhibits apoptosis of monocytes by activating the TLR4/MAPK/ERK signaling pathway (3), we further investigated the effect of GL on this pathway. HMGB1 significantly increased the p-ERK1/2, whereas GL suppressed the HMGB1-induced activation of ERK1/2 (Fig. 5). It has been reported that the protein degradation of Mcl-1 is inhibited by ERK (12). Therefore, activation of the ERK pathway could prevent the protein degradation of Mcl-1. We speculated that HMGB1 may inhibit the degradation of Mcl-1 via the MAPK/ERK pathway, and further antagonize apoptosis. Fig. 6 shows that HMGB1 significantly increased the level of Mcl-1 compared to that in the STS treated group. The level of Mcl-1 increased by HMGB1 was remarkably decreased by the ERK1/2 inhibitor SCH772984 (SCH) (10 nM; Medchemexpress LLC, Princeton, NJ, USA) and GL.

\section{Discussion}

The results of the present study suggested that GL attenuates the HMGB1-induced inflammatory reaction by suppressing the
A
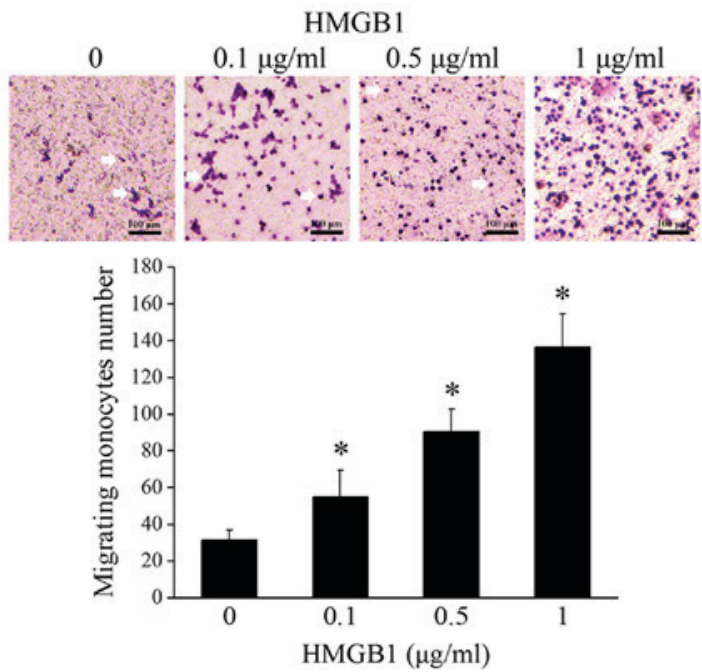

B
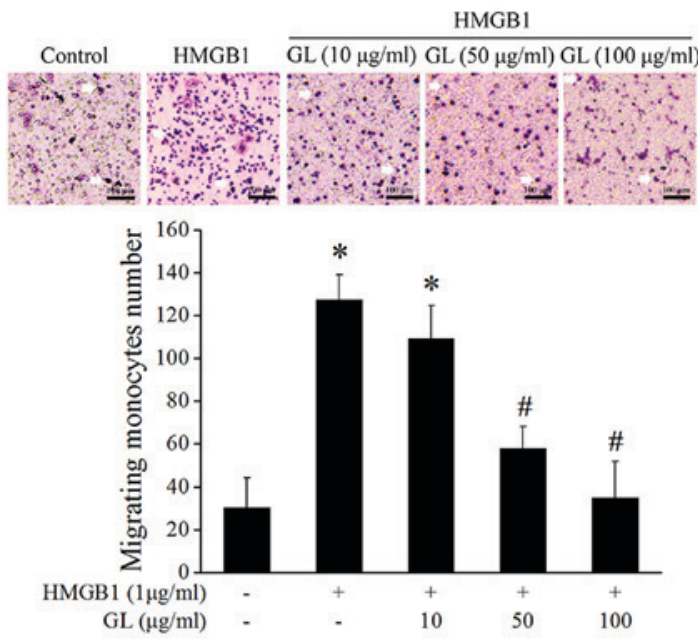

Figure 1. Transwell assay for the detection of cell migration. (A) THP-1 cells were treated with different concentrations of HMGB1. Cell migration ability was detected by Transwell assay. (B) THP-1 cells were treated with $1 \mu \mathrm{g} / \mathrm{ml}$ HMGB1 and different concentrations of GL. Cell migration ability was detected by Transwell assay. Arrows indicate migrated THP-1 cells Data are presented as the mean \pm standard deviation of at least three separate experiments. Scale bars, $100 \mu \mathrm{m}$. "P<0.05 vs. non-HMGB1 control group $(0 \mu \mathrm{g} / \mathrm{ml}) ;{ }^{*} \mathrm{P}<0.05 \mathrm{vs}$. HMGB1 monotherapy group. HMGB1, high mobility group box-1 protein; GL, glycyrrhizin.

migration of monocytes and inducing apoptosis. Furthermore, HMGB1 might promote the migration and suppress apoptosis of monocytes through the NF- $\mathrm{\kappa B} / \mathrm{MCP}-1$ and MAPK/ERK/Mcl-1 signaling pathways. Both of these signaling pathways could be blocked by GL treatment.

Consistent with a previous study (3), HMGB1 promoted the migration, an event accompanying the recruitment of monocytes (13), and suppressed the apoptosis of monocytes. We used THP-1 cells as a research model because of its wide application in the study of monocyte function (14). GL treatment suppressed the HMGB1-induced migration of monocytes and anti-apoptosis effects in a dose-dependent manner. The anti-inflammatory effect of GL on monocytes was related to the concentration of GL.

A previous study showed that HMGB1 could activate the RAGE/NF- $\kappa B$ pathway and promote the recruitment of monocytes (3). In addition, HMGB1 suppressed the apoptosis of monocytes by activating the TLR4/MAPK/ERK pathway. The 

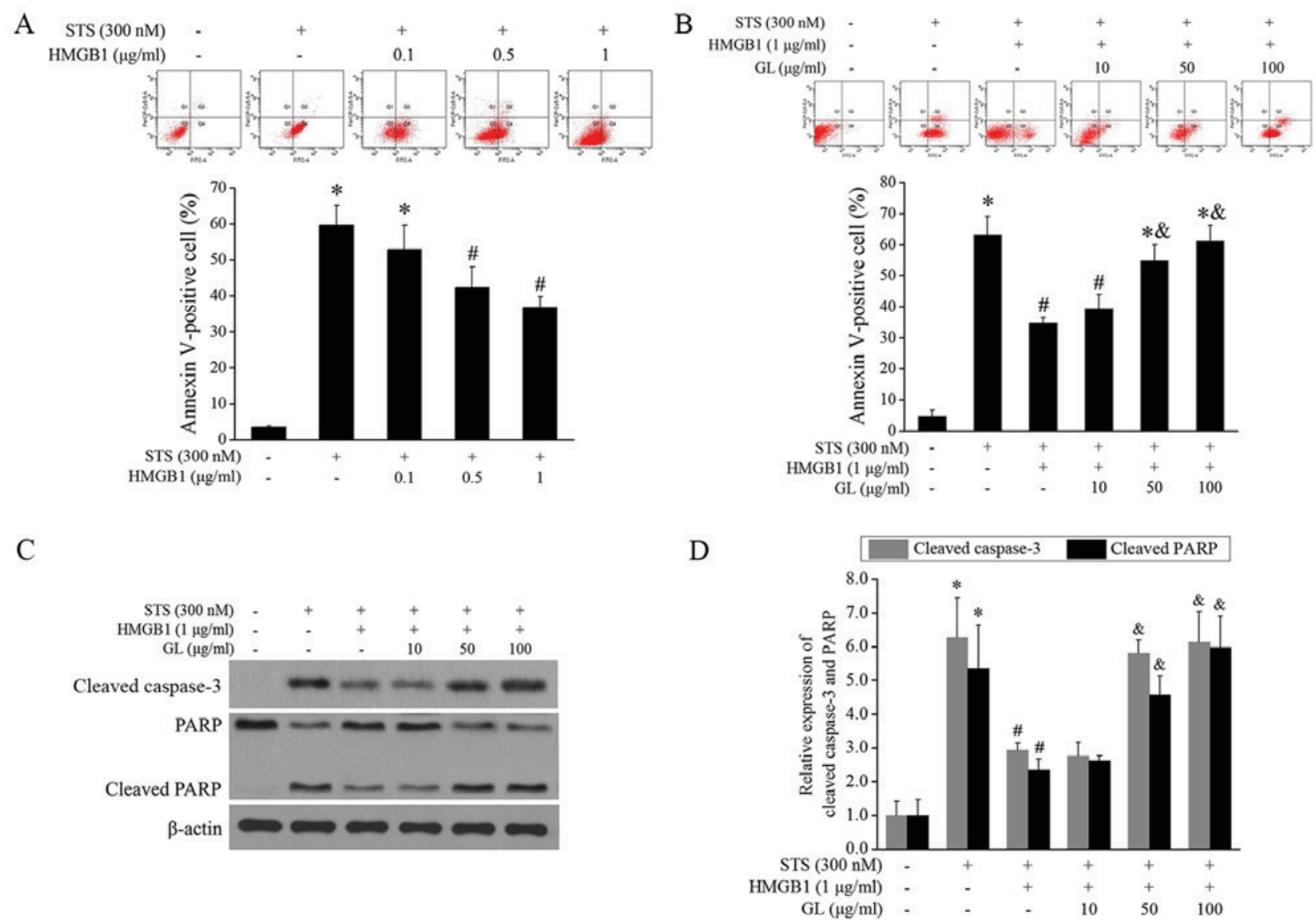

Figure 2. GL treatment increased monocyte apoptosis. (A) THP-1 cells were treated with $300 \mathrm{nM}$ STS and different concentrations of HMGB1. Cell apoptosis was detected by flow cytometry. (B) THP-1 cells were treated with $300 \mathrm{nM} \mathrm{STS}$ and $1 \mu \mathrm{g} / \mathrm{ml}$ HMGB1 or different concentrations of GL. Cell apoptosis was detected by flow cytometry. (C) THP-1 cells were treated with $300 \mathrm{nM} \mathrm{STS}$ and $1 \mu \mathrm{g} / \mathrm{ml}$ HMGB1 or different concentrations of GL. Cleaved caspase-3 and PARP were analyzed by western blotting. (D) Grayscale analysis chart of the relative expression of cleaved caspase-3 and PARP. Data are presented as the mean \pm standard deviation of at least three separate experiments. " $\mathrm{P}<0.05$ vs. control group; ${ }^{*} \mathrm{P}<0.05$ vs. STS monotherapy group; ${ }^{\circledR} \mathrm{P}<0.05$ vs. STS+HMGB1 group. HMGB1, high mobility group box-1 protein; GL, glycyrrhizin; STS, staurosporine; PARP, poly(adenosine diphosphate ribose) polymerase.

A

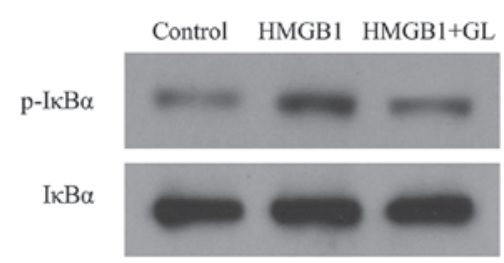

$\mathrm{C}$

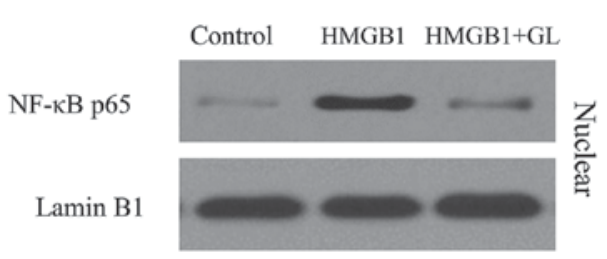

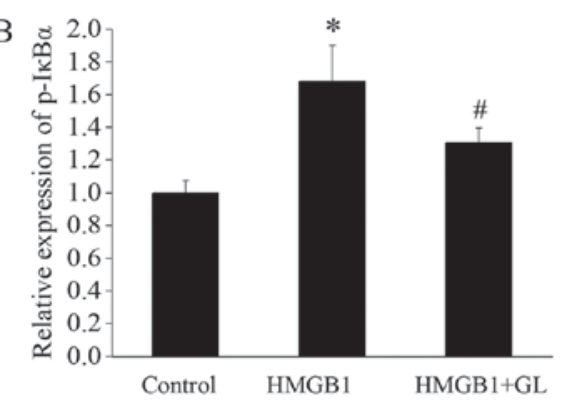

D

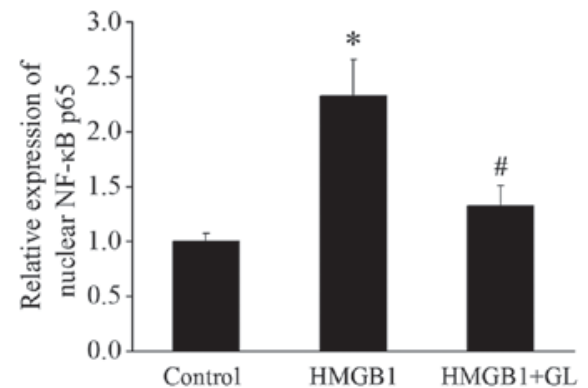

Figure 3. GL treatment inhibited HMGB1-induced activation of NF- $\kappa$ B. THP-1 cells were treated with HMGB1 and GL (100 $\mu \mathrm{g} / \mathrm{ml})$. (A) Western blot analysis of the expression levels of $\mathrm{p}$-ІкB $\alpha$ and IкB $\alpha$. (B) Grayscale analysis chart of the relative expression of p-IкB $\alpha$. (C) Western blot analysis of the expression levels of nuclear NF-kB p65. Lamin B1 was used as a nuclear marker. (D) Grayscale analysis chart of the relative expression of nuclear NF- $\mathrm{kB}$ p65. Data are presented as the mean \pm standard deviation of at least three separate experiments. ${ }^{*}<0.05$ vs. control group; ${ }^{*} \mathrm{P}<0.05$ vs. HMGB1 monotherapy group. HMGB1,

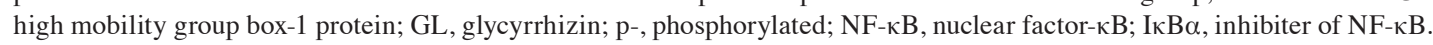




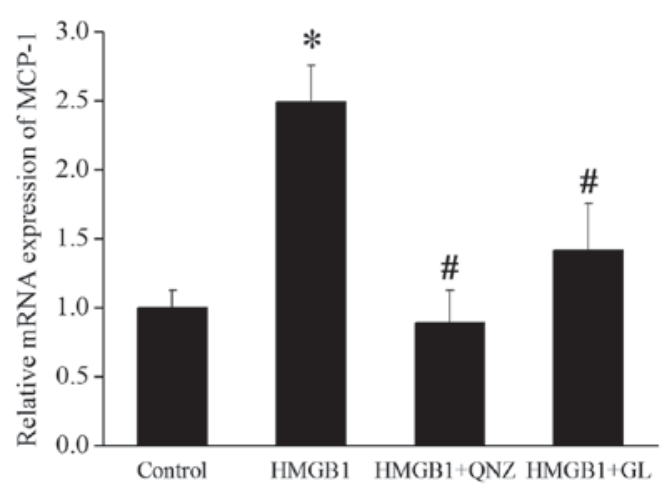

Figure 4. GL treatment downregulated MCP-1. THP-1 cells were treated with HMGB1, the NF- $\mathrm{kB}$ inhibitor QNZ (100 nM) and GL. The expression of MCP-1 was determined by reverse transcription-quantitative polymerase chain reaction. Data are presented as the mean \pm standard deviation of at least three separate experiments. ${ }^{*} \mathrm{P}<0.05$ vs. control group; ${ }^{*} \mathrm{P}<0.05$ vs. HMGB1 monotherapy group. HMGB1, high mobility group box-1 protein; GL,

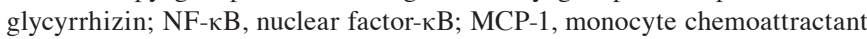
protein-1.

A

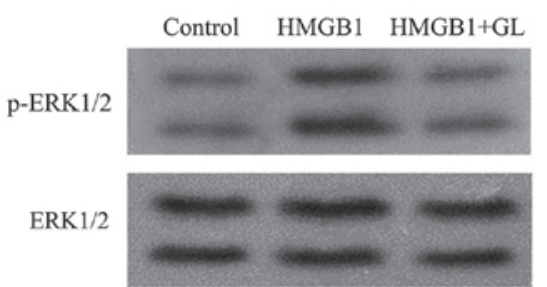

B

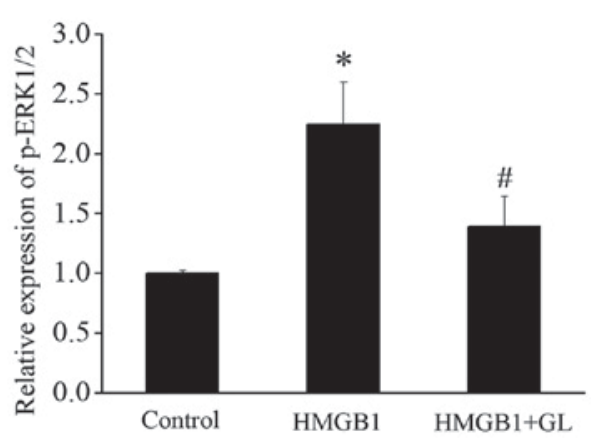

Figure 5. GL treatment inhibited HMGB1-induced activation of ERK (A) THP-1 cells were treated with HMGB1 and GL. The expression levels of p-ERK1/2 and ERK1/2 were detected by western blotting. (B) Grayscale analysis chart of the relative expression of $\mathrm{p}$-ERK1/2. Data are presented as the mean \pm standard deviation of at least three separate experiments. ${ }^{*} \mathrm{P}<0.05$ vs. control group; ${ }^{~} \mathrm{P}<0.05$ vs. HMGB1 monotherapy group. HMGB1, high mobility group box-1 protein; GL, glycyrrhizin; ERK, extracellular signal-regulated kinase; p-, phosphorylated.

cell receptor signaling pathways involved suggested a potential anti-inflammatory mechanism underlying the effect of GL. Our previous study showed that GL inhibits the MAPK/NF-kB pathway by blocking the interaction of HMGB1 with TLR4 and RAGE, as determined by co-immunoprecipitation assays using rat NR8383 alveolar macrophages (8). In the present study in THP-1 cells, GL probably functions through the same signaling pathway in monocytes.

Different properties of monocytes and macrophages affect cell behavior, which is affected by the downstream proteins of signaling pathways. MCP-1 is a small proinflammatory factor and a member of the $\mathrm{CC}$ chemokine family that is secreted by a
A STS $(300 \mathrm{nM})$
HMGB1 $(1 \mu \mathrm{g} / \mathrm{ml})$
SCH $(10 \mathrm{nM})$
GL $(100 \mu \mathrm{g} / \mathrm{ml})$

\section{Mcl-1}

$\beta$-actin

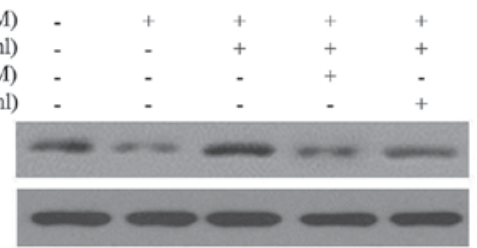

B

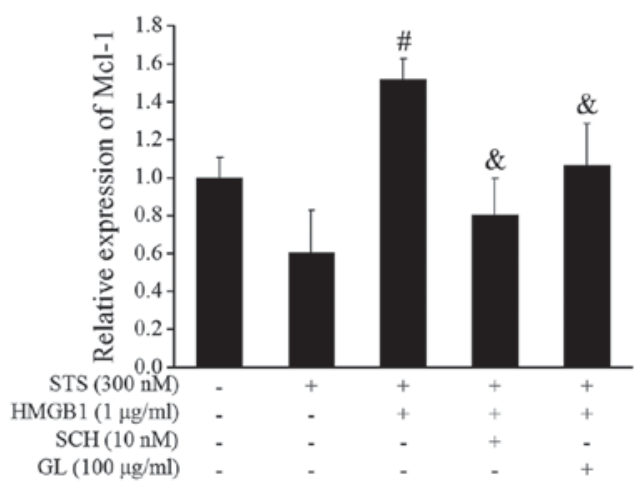

Figure 6. GL treatment decreased the expression of Mcl-1. (A) THP-1 cells were treated with $300 \mathrm{nM}$ STS, $1 \mu \mathrm{g} / \mathrm{ml} \mathrm{HMGB} 1$, the ERK1/2 inhibitor $\mathrm{SCH}(10 \mathrm{nM})$ or $100 \mu \mathrm{g} / \mathrm{ml} \mathrm{GL}$. The expression level of Mcl-1 was detected by western blotting. (B) Grayscale analysis chart of the relative expression of Mcl-1. Data are presented as the mean \pm standard deviation of at least three separate experiments. ${ }^{~} \mathrm{P}<0.05$ vs. STS monotherapy group; ${ }^{\circledR} \mathrm{P}<0.05$ vs. STS+HMGB1 group. HMGB1, high mobility group box-1 protein; GL, glycyrrhizin; ERK, extracellular signal-regulated kinase; Mcl-1, myeloid cell leukemia 1; STS, staurosporine; SCH, SCH772984.

variety of cells, such as epithelial cells, endothelial cells, smooth muscle cells, fibroblasts, and monocytes. Some specific leukocytes including monocytes, lymphocytes, and mast cells can be recruited and activated by MCP-1 (15). A previous study showed that MCP-1 could be produced via the NF- $\mathrm{kB}$ signaling pathway to mediate the migration of amoeboid microglia in rats (11). Our study showed that HMGB1 could increase the expression of MCP-1, whereas both an NF- $\mathrm{BB}$ inhibitor and GL significantly suppressed MCP-1 expression induced by HMGB1. These results indicated that GL might act through the RAGE/NF- $\kappa B$ signaling pathway and suppress the production of MCP-1. Further research is necessary to confirm this hypothesis.

Dysregulation of apoptosis in specific leukocytes may increase the duration and severity of the systemic inflammatory response (16). Anti-apoptotic monocytes release cytokines and cytotoxic products, which recruit additional leukocytes and damage host cells, with the end result of the amplification of the inflammatory cascade. Mcl-1 is a unique member of the Bcl-2 family, which plays an important role in apoptosis. It has been widely investigated in the field of cancer. Because of its anti-apoptotic function, Mcl-1 levels are inversely correlated with neutrophil apoptosis $(17,18)$. Although Mcl-1 has a short half-life of $<5 \mathrm{~h}$, the ERK pathway can delay protein degradation and prolong its lifespan (12), resulting in a decrease in apoptosis. We found that the level of Mcl-1 was significantly increased because of HMGB1, and further remarkably decreased by both the ERK $1 / 2$ inhibitor and GL. This suggested that HMGB1 suppressed the degradation of Mcl-1 via the MAPK/ERK pathway. GL might restore Mcl-1 to normal half-life by blocking this signaling pathway, and promote the recovery of apoptosis in monocytes. This signaling pathway mechanism merits further investigation. 
In conclusion, GL inhibited the effect of HMGB1 on monocyte migration and apoptosis, probably via the $\mathrm{NF}-\kappa \mathrm{B} / \mathrm{MCP}-1$ pathway and ERK/Mcl-1 pathway. GL restored the normal function of monocytes, resulting in the reduction of the systemic inflammatory response.

\section{Acknowledgements}

Not applicable.

\section{Funding}

The present study was supported by a general project from Shanghai Municipal Commission of Health and Family Planning (grant nos. 201540043 and 2016ZB0202-01), and The Scientific Research Project supported by Huashan Hospital, Fudan University (grant no. 2014QD15).

\section{Availability of data and materials}

The analyzed data sets generated during the study are available upon reasonable request for non-commercial purposes, without breaching participant confidentiality.

\section{Authors' contributions}

JT, WW and YG conceived and designed the experiments. JT, FZ and SD performed the experiments, and JT and HZ analyzed the data. JT, WW and YG wrote the paper. YG approved the final version to be published.

\section{Ethics approval and consent to participate}

Not applicable.

\section{Consent for publication}

Not applicable.

\section{Competing interests}

The authors declare that they have no competing interests.

\section{References}

1. Rhodes A, Evans LE, Alhazzani W, Levy MM, Antonelli M, Ferrer R, Kumar A, Sevransky JE, Sprung CL, Nunnally ME, et al: Surviving sepsis campaign: international guidelines for management of sepsis and septic shock: 2016. Crit Care Med 45: 486-552, 2017.
2. Li J, Carr B, Goyal M and Gaieski DF: Sepsis: The inflammatory foundation of pathophysiology and therapy. Hosp Pract (1995) 39: 99-112, 2011.

3. Vogel S, Rath D, Borst O, Mack A, Loughran P, Lotze MT, Neal MD, Billiar TR and Gawaz M: Platelet-derived high-mobility group box 1 promotes recruitment and suppresses apoptosis of monocytes. Biochem Biophys Res Commun 478: 143-148, 2016.

4. Shi JR, Mao LG, Jiang RA, Qian Y, Tang HF and Chen JQ: Monoammonium glycyrrhizinate inhibited the inflammation of LPS-induced acute lung injury in mice. Int Immunopharmacol 10: 1235-1241, 2010.

5. Asl MN and Hosseinzadeh $\mathrm{H}$ : Review of pharmacological effects of Glycyrrhiza sp. and its bioactive compounds. Phytother Res 22: 709-724, 2008.

6. Korenaga M, Hidaka I, Nishina S, Sakai A, Shinozaki A, Gondo T, Furutani T, Kawano H, Sakaida I and Hino K: A glycyrrhizin-containing preparation reduces hepatic steatosis induced by hepatitis $\mathrm{C}$ virus protein and iron in mice. Liver Int 31: 552-560, 2011.

7. Mollica L, De Marchis F, Spitaleri A, Dallacosta C, Pennacchini D, Zamai M, Agresti A, Trisciuoglio L, Musco G and Bianchi ME: Glycyrrhizin binds to high-mobility group box 1 protein and inhibits its cytokine activities. Chem Biol 14: 431-441, 2007.

8. Zhao F, Fang Y, Deng S, Li X, Zhou Y, Gong Y, Zhu H and Wang W: Glycyrrhizin protects rats from sepsis by blocking HMGB1 signaling. Biomed Res Int 2017: 9719647, 2017.

9. Weber GF, Chousterman BG, He S, Fenn AM, Nairz M, Anzai A, Brenner T, Uhle F, Iwamoto Y, Robbins CS, et al: Interleukin-3 amplifies acute inflammation and is a potential therapeutic target in sepsis. Science 347: 1260-1265, 2015.

10. Livak KJ and Schmittgen TD: Analysis of relative gene expression data using real-time quantitative PCR and the 2(-Delta Delta C(T)) method. Methods 25: 402-408, 2001.

11. Deng YY, Lu J, Ling EA and Kaur C: Monocyte chemoattractant protein-1 (MCP-1) produced via NF-kappaB signaling pathway mediates migration of amoeboid microglia in the periventricular white matter in hypoxic neonatal rats. Glia 57: 604-621, 2009.

12. Namgaladze D, Kollas A and Brüne B: Oxidized LDL attenuates apoptosis in monocytic cells by activating ERK signaling. J Lipid Res 49: 58-65, 2008.

13. Gerhardt T and Ley K: Monocyte trafficking across the vessel wall. Cardiovasc Res 107: 321-330, 2015.

14. Qin Z: The use of THP-1 cells as a model for mimicking the function and regulation of monocytes and macrophages in the vasculature. Atherosclerosis 221: 2-11, 2012.

15. Oppenheim JJ, Zachariae CO, Mukaida N and Matsushima K: Properties of the novel proinflammatory supergene 'intercrine' cytokine family. Annu Rev Immunol 9: 617-648, 1991.

16. Jimenez MF, Watson RW, Parodo J, Evans D, Foster D, Steinberg M, Rotstein OD and Marshall JC: Dysregulated expression of neutrophil apoptosis in the systemic inflammatory response syndrome. Arch Surg 132: 1263-1270, 1997.

17. Craig RW: MCL1 provides a window on the role of the BCL2 family in cell proliferation, differentiation and tumorigenesis. Leukemia 16: 444-454, 2002.

18. Leuenroth SJ, Grutkoski PS, Ayala A and Simms HH: The loss of Mcl-1 expression in human polymorphonuclear leukocytes promotes apoptosis. J Leukoc Biol 68: 158-166,2000. 